\title{
Superscars in the Šeba billiard
}

\author{
Pär Kurlberg Henrik Ueberschär
}

June 25, 2015

\begin{abstract}
$\|$
P.K. was partially supported by grants from the Göran Gustafsson Foundation for Research in Natural Sciences and Medicine, and the Swedish Research Council (621-20115498).
\end{abstract}

\begin{abstract}
We consider the Laplacian with a delta potential (also known as a "point scatterer", or "Fermi pseudopotential") on an irrational torus, where the square of the side ratio is diophantine. The eigenfunctions fall into two classes - "old" eigenfunctions $(75 \%)$ of the Laplacian which vanish at the support of the delta potential, and therefore are not affected, and "new" eigenfunctions (25\%) which are affected, and as a result feature a logarithmic singularity at the location of the delta potential.

Within a full density subsequence of the new eigenfunctions we determine all semiclassical measures in the weak coupling regime and show that they are localized along 4 wave vectors in momentum space - we therefore prove the existence of so-called "superscars" as predicted by Bogomolny and Schmit [6].

This result contrasts the phase space equidistribution which is observed for a full density subset of the new eigenfunctions of a point scatterer on a rational torus [15]. Further, in the strong coupling limit we show that a weaker form of localization holds for an essentially full density subsequence of the new eigenvalues; in particular quantum ergodicity does not hold.

We also explain how our results can be modified for rectangles with Dirichlet boundary conditions with a point scatterer in the interior. In this case our results extend previous work of Keating, Marklof and Winn who proved the existence of localized semiclassical measures under a clustering condition on the spectrum of the Laplacian.
\end{abstract}

Keywords. Superscars, Šeba billiard, Semiclassical Measures

Department of Mathematics, KTH Royal Institute of Technology, SE-10044

Stockholm, Sweden; e-mail: kurlberg@ math.kth.se

Laboratoire Paul Painlevé, Université Lille 1, CNRS U.M.R. 852459655 Villeneuve d'Ascq Cedex, France; e-mail: henrik.ueberschar@math.univ-lille1.fr

Mathematics Subject Classification (2010): Primary 81Q50; Secondary 58J50 


\section{Introduction}

In the Quantum Chaos literature the Šeba billiard [18], a delta potential placed inside an irrational rectangular billiard, has attracted considerable attention [20, 21, 22, 23, 19, 24, 5]. Šeba introduced the model to investigate the transition between integrability and chaos in quantum systems and numerical experiments revealed features characteristic of chaotic systems: level repulsion and a Gaussian value distribution of the wave functions - in agreement with Berry's random wave conjecture [1].

The present paper deals with irrational tori having diophantine 3 aspect ratio; for convenience the main focus is on periodic rather than Dirichlet boundary conditions, but the methods also apply in the latter case (cf. Appendix A.)

The eigenfunctions of this system fall into two classes - old and new eigenfunctions. The old eigenfunctions are simply eigenfunctions of the Laplacian which vanish at the position of the scatterer $x_{0}$ and therefore do not feel its presence. In the case of an irrational torus they make up 75\% of the spectrum. In this paper we will only be interested in the new eigenfunctions, which do feel the effect of the scatterer and feature a logarithmic singularity at $x_{0}$. They make up the remaining $25 \%$ of the spectrum.

\subsection{Statement of the main result}

We prove that a full density subsequence of the new eigenfunctions of the point scatterer fail to equidistribute in phase space in the weak coupling limit. Specifically, these eigenfunctions become localized ("scarred", or even "superscarred") in 4 wave vectors in momentum space and we are able to classify all possible semiclassical measures which may arise along this sequence in the weak coupling regime (i.e., fixed self-adjoint extensions). Moreover, in the strong coupling regime (where the self-adjoint extension parameter varies with the eigenvalue) we are able to show a somewhat weaker result, namely that a subsequence of almost full density fails to equidistribute in phase space.

To describe this more precisely, we first introduce semiclassical measures arising from eigenfunctions.

Definition 1.1. Let $\lambda$ be a new eigenvalue of the scatterer and denote by $g_{\lambda}$ the corresponding $L^{2}$-normalized eigenfunction. Let a $\in C^{\infty}\left(S^{*} \mathbb{T}^{2}\right)$ be a classical observable and let $O p(a)$ be a zeroth order pseudo-differential operator 4 associated with $a$. We define the distribution $d \mu_{\lambda}$ by the identity

$$
\left\langle O p(a) g_{\lambda}, g_{\lambda}\right\rangle=\int_{S^{*} \mathbb{T}^{2}} a d \mu_{\lambda}
$$

\footnotetext{
${ }^{3}$ An irrational $\gamma$ is diophantine if there exist constants $C>0, k \geq 2$ such that $|\gamma-p / q|>C q^{-k}$ for any rational $p / q$.

${ }^{4}$ We will give a precise definition of our choice of quantization in section 2.2
} 
By the semiclassical measures for a certain sequence $\left\{\lambda_{n}\right\}$ we mean the limit points of $\left\{d \mu_{\lambda_{n}}\right\}$ in the weak-* topology.

By a subsequence of full density we mean the following.

Definition 1.2. Let $\mathcal{S} \subset \mathbb{R}$ be a countably infinite sequence of increasing numbers which accumulate at infinity. We say that $\mathcal{S}^{\prime} \subset \mathcal{S}$ is a subsequence of full density if

$$
\lim _{X \rightarrow \infty} \frac{\#\left\{x \in \mathcal{S}^{\prime} \mid x \leq X\right\}}{\#\{x \in \mathcal{S} \mid x \leq X\}}=1
$$

The following definitions will be used throughout the paper.

Definition 1.3. For $a>0$ a fixed real number, define a lattice

$$
\mathcal{L}_{0}:=\mathbb{Z}(a, 0) \oplus \mathbb{Z}(0,1 / a) \subset \mathbb{R}^{2}
$$

let $\mathcal{L}$ denote the dual lattice of $\mathcal{L}_{0}$, and let $\mathcal{N}$ denote the set of distinct Laplacian eigenvalues (i.e., squares of norms of the lattice vectors in $\mathcal{L}$.) Further, given $m \in \mathcal{N}$, denote by $\lambda_{m}<m$ the new eigenvalue of the scatterer associated with $m$.

We remark that $\left\{\lambda_{m}: m \in \mathcal{N}\right\}$ is the full set of new eigenvalues; this is due to a certain interlacing property, see Section 2.1 for more details. With notations as above, the main result of this paper is the following result, valid for the weak coupling limit.

Theorem 1.4. Assume that $a^{4} \notin \mathbb{Q}$ is diophantine, and consider the point scatterer perturbation of the Laplacian on the flat torus $\mathbb{T}^{2}=\mathbb{R}^{2} / 2 \pi \mathcal{L}_{0}$. There exists a subsequence $\mathcal{N}^{\prime} \subset \mathcal{N}$ of full density such that the set of semiclassical measures of the sequence $d \mu_{\lambda_{m}}$, $m \in \mathcal{N}^{\prime}$, is given by the following subset of the set of probability measures on the unit cotangent bundle $S^{*} \mathbb{T}^{2}$ :

$$
\mathcal{Q}=\left\{\frac{d x}{\operatorname{vol}\left(\mathbb{T}^{2}\right)} \times \frac{1}{4}\left(\delta_{\theta}+\delta_{-\theta}+\delta_{\pi-\theta}+\delta_{\pi+\theta}\right)(\phi) \frac{d \phi}{2 \pi} \mid \theta \in[0, \pi / 2]\right\} .
$$

Remark 1. As already mentioned, the result can be extended to irrational (diophantine) rectangles with Dirichlet boundary conditions and a delta potential in the interior - the original setting of Šeba's paper. In the appendix to this paper we illustrate how our proof can be modified. For a generic position of the scatterer we prove scarring for a proportion $1-\epsilon$ of all eigenfunctions, for any $\epsilon>0$. In the non-generic case of positions with rational coordinates, a positive proportion of the eigenfunctions do not feel the effect of the scatterer, hence are old Laplacian eigenfunctions. However, our theorem still applies to the new eigenfunctions associated with the remaining part of the spectrum.

In the strong coupling limit, which is studied in the physics literature, and in which features such as level repulsion between the new eigenvalues are observed, we are able to prove the following somewhat weaker result (see Section 4 for its proof.) 
Theorem 1.5. Given $\delta>0$ there exists a subsequence of the new spectrum, of density at least $1-\delta$, on which the momentum representation of the new eigenfunctions carries positive mass on a finite number of points 5 . For $\delta$ fixed, the mass is uniformly bounded from below, and the number of points is uniformly bounded from above.

These results may also be easily modified for rectangular domains with Dirichlet or Neumann boundary conditions (cf. Remark 3 in the appendix.)

\subsection{Discussion}

The scarring phenomenon described above contrasts the equidistribution of a full density subset of new eigenfunctions for a point scatterer on a square torus, both in the weak as well as strong coupling limits (cf. [15].) Interestingly, a key feature for obtaining equidistribution for the square torus is that the unperturbed spectrum has unbounded multiplicities (along a generic sequence), whereas in the diophantine aspect ratio case, where the unperturbed spectrum has bounded multiplicities, most eigenfunctions scar strongly.

Moreover, the type of scarring proven here seems quite different from the sequence of scars established by Hassell [11] for the stadium billiard (his construction is based on quasimodes corresponding to a certain sparse sequence of "bouncing ball modes"), or the construction of scars for cat maps with small quantum periods by de Bièvre, Faure and Nonnenmacher [4] (they construct sparse sequences at most half of whose mass is scarred, and a crucial feature in the construction is having essentially maximal spectral multiplicities; note that Bourgain has shown [7] that scarring does not occur for cat maps if multiplicities are just slightly smaller than maximal.) We also mention Kelmer's construction [13] of scars for certain higher dimensional analogues of cat maps; here the existence of invariant rational isotropic subspaces plays a key role.

In the original setting of the Šeba billiard, i.e., for irrational rectangles with Dirichlet boundary conditions and a delta potential in the interior (and in the weak coupling limit), Keating, Marklof and Winn showed [12] that eigenfunctions can scar in momentum space, provided that the unperturbed eigenfunctions are bounded from below at the location of the scatterer, together with a certain clustering assumption on the spectrum of the Laplacian. (The clustering assumption is implied by the Berry-Tabor conjecture, which suggests that the eigenvalues of a generic integrable system behave like points from a Poisson process.) Our proof can easily be modified for this setting.

Our results also show that contrary to the title of Šeba's original paper [18] there is no "wave chaos" with respect to the wave functions of diophantine rectangular quantum billiards (even though chaotic effects, such as level repulsion, appear in the strong coupling regime [5]) - quantum ergodicity fails, both in the weak and strong coupling regimes.

\footnotetext{
${ }^{5}$ We allow these points to depend on the eigenvalue.
} 
Moreover, in the specific setting of Šeba's original paper (weak coupling and Dirichlet boundary conditions), we show that for any $\epsilon>0$ a proportion $1-\epsilon$ of the eigenfunctions are scarred in momentum space and we determine all possible scarred measures explicitly.

\section{Acknowledgements}

We would like to thank Jens Marklof, Stéphane Nonnenmacher and Zeev Rudnick for very helpful discussions about this work. We would also like to thank the anonymous referee for his careful reading of the paper and many suggestions which led to the improvement of this paper.

\section{Background}

This section has the purpose of providing the reader with a brief summary of various results which will be used in the paper.

\subsection{The spectrum of a point scatterer on an irrational torus}

In order to realize the formal operator

$$
-\Delta+\alpha \delta_{x_{0}}, \quad\left(\alpha, x_{0}\right) \in \mathbb{R} \times \mathbb{T}^{2}
$$

we use self-adjoint extension theory. We simply state the most important facts in this section to make the paper as self-contained as possible. For a more detailed discussion of the theory we refer the reader to the introduction and appendix of the paper [16].

Recall that $\mathbb{T}^{2}=\mathbb{R}^{2} / 2 \pi \mathcal{L}_{0}$. We restrict the positive Laplacian $-\Delta$ to the domain

$$
D_{0}=C_{c}^{\infty}\left(\mathbb{T}^{2} \backslash\left\{x_{0}\right\}\right)
$$

of functions which vanish near the position of the scatterer:

$$
H_{0}=-\left.\Delta\right|_{D_{0}}
$$

The operator $H_{0}$ is symmetric, but it fails to be essentially self-adjoint, in fact $H_{0}$ has deficiency indices $(1,1)$. Therefore there exists a one-parameter family of self-adjoint extensions $H_{\varphi}, \varphi \in(-\pi, \pi]$, which are restrictions of the adjoint $H_{0}^{*}$ of the restricted operator to the domain of functions $f \in \operatorname{Dom}\left(H_{0}^{*}\right)$ which satisfy the logarithmic boundary condition

$$
f(x)=C\left(\cos (\varphi / 2) \frac{\log \left|x-x_{0}\right|}{2 \pi}+\sin (\varphi / 2)\right)+o(1)
$$

as $x \rightarrow x_{0}$ for some constant $C \in \mathbb{C}$. The case $\varphi=\pi$ corresponds to $\alpha=0$, i. e. we simply obtain the unrestricted Laplacian in this case. In this paper we will study the 
operators $H_{\varphi}, \varphi \in(-\pi, \pi)$. In the physics literature [20] the operator $H_{\varphi}$ for fixed $\varphi$ is known as the "weak coupling" quantization of the scatterer.

Let us now focus on the special case of an irrational torus $\mathbb{T}^{2}$. This means we take a lattice $\mathcal{L}_{0}$ such that $a^{4} \notin \mathbb{Q}$. The spectrum of the operator $H_{\varphi}$ consists of two parts:

(A) Eigenfunctions which vanish at $x_{0}$ and therefore do not "feel" the scatterer. These are simply eigenfunctions of the Laplacian, and occur with multiplicity $m-1$ where $m$ is the multiplicity of the corresponding eigenspace of the Laplacian. The multiplicity of the positive old eigenvalues is 3 , unless the corresponding lattice vector lies on one of the axes, in which case it is 1 .

(B) Eigenfunctions which feature a logarithmic singularity at $x_{0}$. These "feel" the effect of the scatterer, and turn out to be given by the Green's functions $G_{\lambda}=$ $(\Delta+\lambda)^{-1} \delta_{x_{0}}$. The new eigenvalues $\lambda$ occur with multiplicity 1 and interlace with the "old" Laplace eigenvalues (counted without multiplicity.)

We will be interested in the eigenfunctions of type (B), and in particular we will study how these eigenfunctions are distributed in phase space as the eigenvalue tends to infinity. Recall that $\mathcal{N}$ denotes the set of distinct eigenvalues of the Laplacian on $\mathbb{T}^{2}$ (these are just norms squared of the lattice vectors in $\mathcal{L}$ ). For given $n \in \mathcal{N}$ denote its multiplicity by $r(n)$.

The eigenvalues of type (B) are solutions to the equation

$$
\sum_{n \in \mathcal{N}} r(n)\left(\frac{1}{n-\lambda}-\frac{n}{n^{2}+1}\right)=\tan (\varphi / 2) \sum_{n \in \mathcal{N}} \frac{r(n)}{n^{2}+1}
$$

and they interlace with the distinct Laplacian eigenvalues

$$
\mathcal{N}=\left\{0=n_{0}<n_{1}<n_{2}<\cdots\right\}
$$

as follows

$$
\lambda_{n_{0}}<0=n_{0}<\lambda_{n_{1}}<n_{1}<\lambda_{n_{2}}<n_{2}<\cdots
$$

where the new eigenvalue associated with $n \in \mathcal{N}$ is denoted by $\lambda_{n}$.

\subsection{Quantization of phase space observables}

Recall that $\mathbb{T}^{2}=\mathbb{R}^{2} / 2 \pi \mathcal{L}_{0}$ where $\mathcal{L}_{0}=\mathbb{Z}(a, 0) \oplus \mathbb{Z}(0,1 / a), a>0$, and $\mathcal{L}$ denotes its dual lattice. Consider a classical symbol $a \in C^{\infty}\left(S^{*} \mathbb{T}^{2}\right)$, where $S^{*} \mathbb{T}^{2} \simeq \mathbb{T}^{2} \times S^{1}$ denotes 
the unit cotangent bundle of $\mathbb{T}^{2}$. We may expand $a$ in the Fourier series

$$
a(x, \phi)=\sum_{\zeta \in \mathcal{L}, k \in \mathbb{Z}} \hat{a}(\zeta, k) e^{\mathrm{i}\langle\zeta, x\rangle+\mathrm{i} k \phi} .
$$

We choose the following quantization of the symbol $a$. Let $f \in L^{2}\left(\mathbb{T}^{2}\right)$ with Fourier expansion

$$
f(x)=\sum_{\xi \in \mathcal{L}} \hat{f}(\xi) e^{\mathrm{i}\langle\xi, x\rangle} .
$$

On the Fourier side the action of the 0 -th order pseudodifferential operator $O p(a)$ is defined by (we have chosen a "right" quantization, which means we first apply momentum then position operators (cf. section 2.1, in [15]))

$$
(\widehat{O p(a) f})(\xi)=\sum_{\zeta \neq \xi \in \mathcal{L}, k \in \mathbb{Z}} \hat{a}(\zeta, k)\left(\frac{\tilde{\xi}-\tilde{\zeta}}{|\xi-\zeta|}\right)^{k} \hat{f}(\xi-\zeta)+\sum_{k \in \mathbb{Z}} \hat{a}(\xi, k) \hat{f}(0)
$$

where for a given $\xi=\left(\xi_{1}, \xi_{2}\right) \in \mathcal{L}$ we define $\tilde{\xi}:=\xi_{1}+\mathrm{i} \xi_{2}$.

In terms of the Fourier coefficients the matrix elements of $O p(a)$ can be written as

$$
\langle O p(a) f, f\rangle=\sum_{\xi \in \mathcal{L}}(\widehat{O p(a) f})(\xi) \overline{\hat{f}(\xi)}
$$

With $e_{\zeta, k}(x, \phi):=e^{\mathrm{i}\langle\zeta, x\rangle+\mathrm{i} k \phi}$, we then have

$$
\left\langle O p\left(e_{\zeta, k}\right) f, f\right\rangle=\sum_{\xi \in \mathcal{L} \backslash\{\zeta\}}\left(\frac{\tilde{\xi}-\tilde{\zeta}}{|\xi-\zeta|}\right)^{k} \overline{\hat{f}(\xi)} \hat{f}(\xi-\zeta)+\overline{\hat{f}(\zeta)} \hat{f}(0) .
$$

\subsubsection{Mixed modes}

If $\zeta \neq 0$ we have the bound

$$
\left|\left\langle O p\left(e_{\zeta, k}\right) f, f\right\rangle\right| \leq \sum_{\xi \in \mathcal{L}}|\hat{f}(\xi)||\hat{f}(\xi-\zeta)| .
$$

In the case $f=g_{\lambda}=G_{\lambda} /\left\|G_{\lambda}\right\|_{2}$ we have the $L^{2}$-expansion

$$
G_{\lambda}\left(x, x_{0}\right)=-\frac{1}{4 \pi^{2}} \sum_{\xi \in \mathcal{L}} c(\xi) e^{\mathrm{i}\left\langle x-x_{0}, \xi\right\rangle}
$$

where $c(\xi)=\frac{1}{|\xi|^{2}-\lambda}$. We obtain

$$
\left|\left\langle O p\left(e_{\zeta, k}\right) g_{\lambda}, g_{\lambda}\right\rangle\right| \leq \frac{\sum_{\xi \in \mathcal{L}}|c(\xi)||c(\xi-\zeta)|}{\sum_{\xi \in \mathcal{L}}|c(\xi)|^{2}}
$$

In [16] it was proved that one can construct a full density subsequence $\mathcal{N}^{\prime} \subset \mathcal{N}$ such that for any nonzero lattice vector $\zeta \in \mathcal{L}$ the matrix elements of $O p\left(e_{\zeta, k}\right)$ vanish as $n \rightarrow \infty$ along $\mathcal{N}^{\prime}$. The following result was obtained. 
Theorem 2.1. (Rudnick-U., 2012) Let $\mathcal{L}$ be a unimodular lattice as above. There exists a subsequence $\mathcal{N}^{\prime} \subset \mathcal{N}$ of full density such that for any $\zeta \in \mathcal{L}, \zeta \neq 0, k \in \mathbb{Z}$

$$
\lim _{\substack{n \rightarrow \infty \\ n \in \mathcal{N}^{\prime}}}\left\langle O p\left(e_{\zeta, k}\right) g_{\lambda_{n}}, g_{\lambda_{n}}\right\rangle=0 .
$$

Remark 2. Although the paper [16] is solely concerned with the weak coupling regime, i.e. fixed self-adjoint extensions, the theorem holds generally for Green's functions $G_{\lambda_{n}}$ and any sequence of real numbers $\left\{\lambda_{n}\right\}$ which interlaces with the Laplacian eigenvalues on $\mathbb{T}^{2}$. A detailed explanation is given in [15], p. 7, Remark 3.

\subsubsection{Pure momentum modes}

Let us consider the case $\zeta=0$. We rewrite the matrix elements as

$$
\begin{aligned}
\left\langle O p\left(e_{0, k}\right) g_{\lambda}, g_{\lambda}\right\rangle & =\frac{\sum_{\xi \in \mathcal{L} \backslash\{0\}}(\tilde{\xi} /|\xi|)^{k}|c(\xi)|^{2}+|c(0)|^{2}}{\sum_{\xi \in \mathcal{L}}|c(\xi)|^{2}} \\
& =\frac{\sum_{n \in \mathcal{N}} \frac{w_{k}(n)}{(n-\lambda)^{2}}}{\sum_{n \in \mathcal{N}} \frac{r(n)}{(n-\lambda)^{2}}}
\end{aligned}
$$

where for $0 \neq n \in \mathcal{N}$ we define the exponential sum

$$
w_{k}(n):=\sum_{\substack{|\xi|^{2}=n \\ \xi \in \mathcal{L} \backslash\{0\}}}\left(\frac{\tilde{\xi}}{|\xi|}\right)^{k},
$$

and for notational convenience we set $w_{k}(0):=1$.

\subsection{Pair correlations for values of quadratic forms}

In this section we will briefly review a result of Eskin, Margulis and Mozes [9] on the pair correlations of the values of the quadratic form $Q(k, l)=a^{-2} k^{2}+a^{2} l^{2}, k, l \in \mathbb{Z}$, where $\gamma=a^{4}$ is diophantine. We have the following theorem (cf. Thm. 1.7 in [9]), which we only state in the special case relevant to the present paper. Note that area $\left(\mathbb{T}^{2}\right) / 4 \pi=\pi$.

Theorem 2.2. (Eskin-Margulis-Mozes, 2005) Let $\gamma=a^{4}$ be diophantine and $0 \notin(b, c)$. Denote the Laplacian eigenvalues on $\mathbb{T}^{2}=\mathbb{R}^{2} / 2 \pi \mathcal{L}_{0}$ by $\left\{\lambda_{j}\left(\mathbb{T}^{2}\right)\right\}$. Then

$$
\lim _{X \rightarrow \infty} \frac{\#\left\{\lambda_{j}\left(\mathbb{T}^{2}\right), \lambda_{k}\left(\mathbb{T}^{2}\right) \leq X \mid \lambda_{j}\left(\mathbb{T}^{2}\right)-\lambda_{k}\left(\mathbb{T}^{2}\right) \in(b, c)\right\}}{X}=\pi^{2}(c-b) .
$$

The theorem above proves the Berry-Tabor conjecture [2] for the pair correlations of the Laplacian eigenvalues on the torus $\mathbb{T}^{2}$, where $\gamma=a^{4}$ is diophantine. Recall that the Laplacian eigenvalues are given by the squared norms $\left(k^{2}+a^{4} l^{2}\right) / a^{2}$ and the ordered set of such distinct squared norms is denoted by $\mathcal{N}$. 
As in the irrational case the multiplicities of the Laplacian eigenvalues on the torus are generically 4 , we have for the pair correlation of the distinct Laplacian eigenvalues, i.e. the set of norms $\mathcal{N}$

$$
\lim _{X \rightarrow \infty} \frac{\{m, n \in \mathcal{N} \mid m, n \leq X, m-n \in(b, c)\}}{X}=\frac{\pi^{2}}{16}(c-b) .
$$

Letting

$$
\mathcal{N}(X):=\{n \in \mathcal{N} \mid n \leq X\}
$$

denote the intersection of $\mathcal{N}$ and the interval $[0, X]$, we have the counting asymptotic ("Weyl's law")

$$
|\mathcal{N}(X)| \sim \frac{\pi}{4} X
$$

as $X \rightarrow \infty$. Consequently, we obtain

$$
\lim _{X \rightarrow \infty} \frac{1}{|\mathcal{N}(X)|} \#\{m, n \in \mathcal{N}(X) \mid m-n \in(b, c)\}=\frac{\pi}{4}(c-b) .
$$

We note that the mean spacing is $4 / \pi$ (cf. eq. (2.15)).

\section{The weak coupling limit - proof of Theorem 1.4}

We begin by proving the following proposition.

Proposition 3.1. Let $\mathcal{L}$ be a diophantine rectangular unimodular lattice as above6. There exists a subsequence $\mathcal{N}^{\prime} \subset \mathcal{N}$ of full density such that for $m \in \mathcal{N}^{\prime}$ and any integer $k$,

$$
\left\langle O p\left(e_{0, k}\right) g_{\lambda_{m}}, g_{\lambda_{m}}\right\rangle=\frac{w_{k}(m)}{r(m)}+o(1)
$$

as $m \rightarrow \infty$ along $\mathcal{N}^{\prime}$.

Before giving the proof of Proposition 3.1 we recall the following bound from [17]; it shows that, in the weak coupling regime, the new eigenvalues of the scatterer and the eigenvalues of the Laplacian generically "clump" together.

Theorem 3.2. Let $\mathcal{L}$ be an irrational lattice as above. Given any increasing function $f$ such that $f(m) \rightarrow \infty$ as $m \rightarrow \infty$ along $\mathcal{N}$ there exists a density one subsequence $\mathcal{N}^{\prime \prime} \subset \mathcal{N}$ such that for all $m \in \mathcal{N}^{\prime \prime}$,

$$
0<m-\lambda_{m} \ll \frac{f(m)}{\log m}
$$

The following key Lemma will allow us to "circumvent" the lack of uniformity in the size of the interval $(b, c)$ in Theorem 2.2 .

\footnotetext{
${ }^{6}$ In particular, $a^{4} \notin \mathbb{Q}$ is diophantine; cf. Definition 1.3
} 
Lemma 3.3. For $A \geq 3$ we have

$$
\sum_{\substack{m, n \in \mathcal{N}(x) \\|m-n|>A}} \frac{1}{|m-n|^{2}} \ll \frac{x}{A}
$$

Proof. Given an integer $k \geq 0$, define

$$
M(k):=|\{n \in \mathcal{N}: n \in[k, k+1]\}|
$$

We begin by deducing an $L^{2}$ bound on $M(k)$ using Theorem 2.2 ,

$$
\begin{aligned}
\sum_{k \leq T} M(k)^{2}=\sum_{k \leq T} \mid\{m, n \in \mathcal{N}: & m, n \in[k, k+1]\} \mid \\
& \leq|\{m, n \in \mathcal{N}: m, n \leq T+1, m-n \in[-1,1]\}|
\end{aligned}
$$

which, by Theorem 2.2 , is

$$
\ll 2(T+1)+(T+1) \ll T
$$

(note that we include pairs $m, n \leq T+1$ for which $m=n$; this gives rise to the additional $T+1$ term.)

Using Cauchy-Schwarz, we now find that for $l \ll T$, we have

$$
\sum_{k \leq T} M(k) M(k+l) \leq\left(\sum_{k \leq T} M(k)^{2}\right)^{1 / 2} \cdot\left(\sum_{k \leq T+l} M(k)^{2}\right)^{1 / 2} \ll T^{1 / 2} \cdot T^{1 / 2}=T
$$

We may now conclude the proof:

$$
\begin{gathered}
\sum_{\substack{m, n \in \mathcal{N}(x) \\
|m-n|>A}} \frac{1}{|m-n|^{2}} \ll \sum_{k=A}^{x} \frac{\mid\{m, n \in \mathcal{N}(x): m<n, n-m \in[k, k+1]\}}{k^{2}} \\
\leq \sum_{k=A}^{x} \frac{1}{k^{2}} \sum_{l \leq x} M(l) \cdot(M(l+k)+M(l+k+1))
\end{gathered}
$$

which, by using (3.3), is

$$
\ll \sum_{k=A}^{x} \frac{x}{k^{2}} \ll \frac{x}{A}
$$

We can now prove the following key estimate.

Proposition 3.4. There exists a subsequence $\mathcal{N}_{1} \subset \mathcal{N}$ of full density such that for $m \in \mathcal{N}_{1}$

$$
\sum_{n \in \mathcal{N}, n \neq m} \frac{1}{(n-m)^{2}}=O\left((\log m)^{2-\epsilon}\right) .
$$


Proof. Let $G(m)=(\log m)^{-1+\epsilon}$ for a small fixed $\epsilon \in(0,1)$, and denote by $m_{-}, m_{+}$the nearest neighbours to the left and right of $m \in \mathcal{N}$. We claim that the subsequence

$$
\mathcal{N}_{0}=\left\{m \in \mathcal{N}|| m-m_{-}|,| m-m_{+} \mid \geq G(m)\right\}
$$

is of full density in $\mathcal{N}$. Let us assume for a contradiction that the sequence

$$
\mathcal{N}_{0}^{\prime}=\left\{m \in \mathcal{N}|| m-m_{-} \mid \text {or }\left|m-m_{+}\right|<G(m)\right\}
$$

is of non-zero density, i.e., that for some $\eta>0$,

$$
\left|\mathcal{N}_{0}^{\prime}(x)\right| \geq \eta|\mathcal{N}(x)|
$$

holds for a sequence of values of $x$ tending to infinity. Recall that $N(x) \sim \frac{\pi}{4} x$, as $x \rightarrow \infty$, since area $\left(\mathbb{T}^{2}\right)=4 \pi^{2}$ and the multiplicity is generically 4 . Using Theorem 2.2 , we thus find that as $x \rightarrow \infty$,

$$
\begin{aligned}
& \frac{1}{|\mathcal{N}(x)|} \#\left\{m \in \mathcal{N}_{0}^{\prime} \mid m \leq x\right\} \\
\leq & \frac{1}{|\mathcal{N}(x)|} \#\{m, n \in \mathcal{N}|| m-n \mid<G(m), \quad m \neq n, \quad m, n \leq x\} \\
\leq & \frac{1}{|\mathcal{N}(x)|} \#\left\{m, n \in \mathcal{N}|| m-n \mid \leq \frac{\eta}{\pi}, \quad m \neq n, \quad x^{1 / 4} \leq m, n \leq x\right\} \\
\rightarrow & \frac{\eta}{2} \quad+O\left(x^{-1 / 2}\right)
\end{aligned}
$$

which leads to a contradiction to eq. (3.5).

Next we estimate the sum on the LHS of (3.4). We first note that for $m \in \mathcal{N}(x)$ and $x$ large we have

$$
\begin{aligned}
\sum_{n \in \mathcal{N}, n \neq m} \frac{1}{(m-n)^{2}} & =\sum_{n \in \mathcal{N}(2 x), n \neq m} \frac{1}{(m-n)^{2}}+\sum_{n \in \mathcal{N}, n>2 x, n \neq m} \frac{1}{(m-n)^{2}} \\
& =\sum_{n \in \mathcal{N}(2 x), n \neq m} \frac{1}{(m-n)^{2}}+O\left(\frac{1}{x}\right)
\end{aligned}
$$

where we used in the last line that $m \leq x$ and $n>2 x$, hence $n-m>n / 2$ and the bound on the second sum follows from Weyl's law (see (2.15) ) and partial summation.

Next we show that there exists a density one subsequence $\mathcal{N}_{1} \subset \mathcal{N}_{0}$ such that for all $m \in \mathcal{N}_{1}(x)$

$$
\sum_{n \in \mathcal{N}(2 x), n \neq m} \frac{1}{(m-n)^{2}} \ll(\log m)^{2-\epsilon}
$$


We have

$$
\begin{aligned}
\sum_{m \in \mathcal{N}_{0}(x)} \sum_{n \in \mathcal{N}(2 x)} \frac{1}{(m-n)^{2}} \leq \sum_{m \in \mathcal{N}(x)} \sum_{\substack{n \in \mathcal{N}(2 x) \\
|n-m| \geq G(m)}} \frac{1}{(m-n)^{2}} \\
=\sum_{m \in \mathcal{N}(x)} \sum_{\substack{n \in \mathcal{N}(2 x) \\
|n-m| \in[G(m), 1]}} \frac{1}{(m-n)^{2}}+\sum_{m \in \mathcal{N}(x)} \sum_{\substack{n \in \mathcal{N}(2 x) \\
|n-m|>1}} \frac{1}{(m-n)^{2}}
\end{aligned}
$$

We estimate the second sum by

$$
\begin{aligned}
& \sum_{m \in \mathcal{N}(x)} \sum_{\substack{n \in \mathcal{N}(2 x) \\
|n-m|>1}} \frac{1}{(m-n)^{2}} \\
\leq & \sum_{k \leq 2 x} \frac{1}{k^{2}} \#\{m \in \mathcal{N}(x), n \in \mathcal{N}(2 x)|| m-n \mid \in[k, k+1)\} \\
= & : \sum_{k \leq 2 x} \frac{c(k)}{k^{2}} \ll \log x
\end{aligned}
$$

where the logarithmic bound follows from

$$
\sum_{k \leq 2 x} c(k) \leq|\{m, n \in \mathcal{N}(2 x):|m-n| \leq 2 x+2\}| \leq|\mathcal{N}(2 x)|^{2} \ll x^{2}
$$

together with summation by parts.

For the first sum we have, by Theorem 2.2 ,

$$
\begin{aligned}
& \sum_{m \in \mathcal{N}(x)} \sum_{\substack{n \in \mathcal{N}(2 x) \\
|n-m| \in[G(m), 1]}} \frac{1}{(m-n)^{2}} \\
\ll & \frac{1}{G(x)^{2}} \#\{m \in \mathcal{N}(x), n \in \mathcal{N}(2 x)|| m-n \mid \in(0,1]\} \\
\ll & \frac{x}{G(x)^{2}} \ll x(\log x)^{2-2 \epsilon} .
\end{aligned}
$$

Now, let

$$
F(m)=\sum_{\substack{n \in \mathcal{N}(2 x) \\|n-m| \geq G(m)}} \frac{1}{(m-n)^{2}} .
$$

From the estimates above we have, for fixed $\delta \in(0,1)$,

$$
\sum_{m \in \mathcal{N}_{0}(x), m \geq x^{\delta}} F(m) \ll x(\log x)^{2-2 \epsilon}
$$

Letting $T(m)=(\log m)^{2-\epsilon}$ and using Chebyshev's inequality we find that

$$
\begin{aligned}
\#\left\{m \in \mathcal{N}_{0}(x) \mid F(m)\right. & \left.\geq T(m), m \geq x^{\delta}\right\} \\
& \ll T(x)^{-1} \sum_{m \in \mathcal{N}_{0}(x), m \geq x^{\delta}} F(m) \ll x(\log x)^{2-2 \epsilon} / T(x)=x /(\log x)^{\epsilon}
\end{aligned}
$$


where we have used that $T(m) \asymp T(x)$ for $m \in\left[x^{\delta}, x\right]$. It follows that $F(m)<T(m)$ is a density one condition inside $\mathcal{N}_{0}(x)$ thereby concluding the proof.

\subsection{Proof of Proposition 3.1}

Proof. Fix some integer $k \neq 0$. In order to construct the full density subsequence we will use the result about "clumping" of the spectrum as stated in Theorem 3.2 . In what follows assume that $f$ is as in Theorem 3.2. By Theorem 3.2, we have for $m \in \mathcal{N}^{\prime \prime}$ that $\left|m-\lambda_{m}\right|^{2}=O\left(f(m)^{2} / \log ^{2} m\right)$, and hence

$$
\left\langle O p\left(e_{0, k}\right) g_{\lambda}, g_{\lambda}\right\rangle=\frac{w_{k}(m)+O\left((\log m)^{-2} f(m)^{2}\right) \sum_{\substack{n \neq m \\ n \in \mathcal{N}}} w_{k}(n)\left(n-\lambda_{m}\right)^{-2}}{r(m)+O\left((\log m)^{-2} f(m)^{2}\right) \sum_{\substack{n \neq m \\ n \in \mathcal{N}}} r(n)\left(n-\lambda_{m}\right)^{-2}}
$$

Let $\mathcal{N}^{\prime}=\mathcal{N}_{1} \cap \mathcal{N}^{\prime \prime}$, with $\mathcal{N}_{1}$ as in Proposition 3.4. For $m \in \mathcal{N}^{\prime}$, by the proof of Proposition 3.4 (in particular note that $\left|m-m_{-}\right|,\left|m-m_{+}\right| \geq G(m)=(\log m)^{-1+\epsilon}$ holds for $m \in \mathcal{N}_{1} \subset \mathcal{N}_{0}$ ), if we take $f(m)=\log \log m$, then

$$
\sum_{\substack{n \neq m \\ n \in \mathcal{N}}} \frac{\left|w_{k}(n)\right|}{\left(n-\lambda_{m}\right)^{2}} \ll \sum_{\substack{n \neq m, n \in \mathcal{N} \\|n-m| \geq G(m)}} \frac{1}{(n-m)^{2}}=O\left((\log m)^{2-\epsilon}\right)
$$

and it follows that

$$
\begin{aligned}
\left\langle O p\left(e_{0, k}\right) g_{\lambda}, g_{\lambda}\right\rangle=\frac{w_{k}(m)+O\left((\log m)^{-\epsilon}\right) \cdot f(m)^{2}}{r(m)+O\left((\log m)^{-\epsilon}\right) \cdot f(m)^{2}}=\frac{w_{k}(m)+o(1)}{r(m)+o(1)} & \\
& =\frac{w_{k}(m)}{r(m)}+o(1)
\end{aligned}
$$

as $m \rightarrow \infty$ (note that $\left|w_{k}(m)\right| \leq r(m) \leq 4$.) So the identity (3.1) follows.

Proposition 3.1 easily gives a classification of the quantum limits which may arise within the sequence $\mathcal{N}^{\prime}$. We are interested in the sequence $d \mu_{\lambda_{m}}, m \in \tilde{\mathcal{N}}$, where $\tilde{\mathcal{N}}$ denotes the intersection of the subsequences in Theorems 2.1 and 3.2 , so $\tilde{\mathcal{N}}$ is of full density. We would like to determine the quantum limits of this sequence, i.e. the limit points in the weak-* topology.

From Theorem 2.1 we know that along $\tilde{\mathcal{N}}$ the limit measures must be flat, or equidistributed, in position. Moreover, from Proposition 3.1 we also know that the matrix elements of pure momentum observables for the eigenfunction $g_{\lambda_{m}}, m \in \tilde{\mathcal{N}}$, tend to stay away from zero, because for an irrational lattice $\mathcal{L}$ the multiplicity $r(n)$ is bounded. The intuition is that the sequence $d \mu_{\lambda_{m}}, m \in \tilde{\mathcal{N}}$, becomes localized in momentum in the semiclassical limit. Theorem 1.4 determines the set of such localized quantum limits. 


\subsection{Proof of Theorem 1.4}

Consider the classical observable

$$
a=\sum_{\zeta \in \mathcal{L}, k \in \mathbb{Z}} \hat{a}(\zeta, k) e_{\zeta, k}
$$

Let $m \in \tilde{\mathcal{N}}$. By a standard diagonalization argument (see section 4 in [15]) it suffices to prove the result for the trigonometric polynomials

$$
P_{J}=\sum_{\substack{\zeta \in \mathcal{L}, k \in \mathbb{Z} \\|\zeta|,|k| \leq J}} \hat{a}(\zeta, k) e_{\zeta, k}
$$

It follows from (3.1) and Theorem 2.1 that

$$
\left\langle O p\left(P_{J}\right) g_{\lambda_{m}}, g_{\lambda_{m}}\right\rangle=\frac{1}{r(m)} \sum_{2 \mid k} \hat{a}(0, k) w_{k}(m)+o(1)
$$

as $m \rightarrow \infty$. For given $m \in \mathcal{N}$ let $\theta_{m} \in[0, \pi / 2]$ be the phase angle of the lattice point on the upper right arc of the circle $|\xi|^{2}=m$, i.e. $\hat{\xi}=m^{1 / 2} e^{\mathrm{i} \theta_{m}}$ for some $\xi \in \mathcal{L}$. Since $\mathcal{L}$ is irrational we have

$$
\frac{w_{k}(m)}{r(m)}=\left\{\begin{array}{l}
\cos \left(k \theta_{m}\right), \quad 2 \mid k \\
0, \quad \text { otherwise }
\end{array}\right.
$$

We have the following Lemma.

Lemma 3.5. The sequence of angles $\left\{\theta_{m}\right\}_{m \in \tilde{\mathcal{N}}}$ is dense in $[0, \pi / 2]$.

Proof. Let $I \subset[0, \pi / 2]$ be a nonempty open interval. As

$$
\left|\left\{m \in \mathcal{N}(x) \mid \theta_{m} \in I\right\}\right|=\left|\left\{\xi \in \mathcal{L}:|\xi|^{2} \leq x, \xi_{1}, \xi_{2} \geq 0, \operatorname{and} \arctan \left(\xi_{2} / \xi_{1}\right) \in I\right\}\right|,
$$

and the latter can be interpreted as the number of $\mathbb{Z}^{2}$-lattice points inside the intersection of an ellipse with a circular sector, dilated by $\sqrt{x}$, we find that $\left|\left\{m \in \mathcal{N}(x) \mid \theta_{m} \in I\right\}\right| \sim$ $c_{I} \cdot x$ as $x \rightarrow \infty$, for some $c_{I}>0$. Since the interval $I$ can be freely chosen, the result follows.

The set of limit points of the sequence $\left(\left\langle O p\left(P_{J}\right) g_{\lambda_{m}}, g_{\lambda_{m}}\right\rangle\right)_{m \in \tilde{\mathcal{N}}}$ is thus given by

$$
\left\{\sum_{2 \mid k} \cos (k \theta) \hat{a}(0, k) \mid \theta \in[0, \pi / 2]\right\} \text {. }
$$

Now, since for $k$ even,

$$
\cos (k \theta)=\frac{1}{4}\left(e^{\mathrm{i} k \theta}+e^{-\mathrm{i} k \theta}+e^{\mathrm{i} k(\pi+\theta)}+e^{\mathrm{i} k(\pi-\theta)}\right)
$$


we find that all such limit set elements can be rewritten as

$$
\begin{aligned}
\sum_{2 \mid k} \cos (k \theta) \hat{a}(0, k) & =\int_{S^{*} \mathbb{T}^{2}} a(x, \phi) \sum_{2 \mid k} \cos (k \theta) e^{-\mathrm{i} k \phi} \frac{d x d \phi}{\operatorname{vol}\left(S^{*} \mathbb{T}^{2}\right)} \\
& =\int_{S^{*} \mathbb{T}^{2}} a(x, \phi) \frac{d x}{\operatorname{vol}\left(\mathbb{T}^{2}\right)} \times \frac{1}{4}\left(\delta_{\theta}+\delta_{-\theta}+\delta_{\pi+\theta}+\delta_{\pi-\theta}\right)(\phi) \frac{d \phi}{2 \pi}
\end{aligned}
$$

and the proof is concluded.

\section{The strong coupling limit}

Let $\lambda_{m}$ be any interlacing sequence. Then there exists a positive density subsequence $\mathcal{N}^{\prime} \subset \mathcal{N}$ such that $\left\{G_{\lambda_{m}}\right\}_{m \in \mathcal{N}^{\prime}}$ does not equidistribute.

As before, for notational convenience we define $\mathcal{N}(T):=\{n \in \mathcal{N}: n \leq T\}$. Further, let $n_{1}, \ldots, n_{k}, \ldots$ be ordered representatives of the elements in the set $\mathcal{N}$ (i.e., so that $\left.n_{1}<n_{2}<\ldots\right)$, and let $s_{i}:=n_{i+1}-n_{i}$ denote the consecutive spacings.

Lemma 4.1. The number of $i \leq T$ such that $s_{i}>G>0$ is $\leq T / G \cdot(4 / \pi+o(1))$.

Proof. Recalling that $\mathcal{N}(T) \sim T \cdot \pi / 4$, we find that $\sum_{i \leq T} s_{i}=(1+o(1)) \cdot 4 T / \pi$. Since $s_{i} \geq 0$ for all $i$, the statement is an immediate consequence of Chebychev's inequality.

Lemma 4.2. Given $D>0, E \geq 1$,

$$
|\{n \in \mathcal{N}(T):|\mathcal{N}(T) \cap[n-D, n+D]|>E+1\}| \ll \frac{D T}{E} .
$$

Proof. By Theorem 2.2 (see (2.14)),

$$
\begin{aligned}
\sum_{n \in \mathcal{N}(T)}(\mid \mathcal{N}(T) \cap & {[n-D, n+D] \mid-1) } \\
= & |\{n, m \in \mathcal{N}(T): m \neq n,|m-n| \leq D\}| \sim \pi^{2} / 16 \cdot 2 D \cdot T
\end{aligned}
$$

and hence, by Chebychev,

$$
|\{n \in \mathcal{N}(T):|\mathcal{N}(T) \cap[n-D, n+D]|>E+1\}| \ll \frac{D T}{E}
$$

We can now finish the proof. Define $\mathcal{N}^{\prime}$ as follows: for $G$ large take $n \in \mathcal{N}$ such that the gap to the nearest left neighbour is at most $G$; by Lemma 4.1 this sequence has density at least $1-2 / G$. 
By Chebychev's inequality and Lemma 3.3 (note that the Lemma is valid also in the stroung coupling limit) we may chose $F$ sufficiently large so that

$$
\left|\left\{m \in \mathcal{N}(T): \sum_{n \in \mathcal{N}(T):|m-n|>3} \frac{1}{|m-n|^{2}}>F\right\}\right| \leq T / G
$$

remove all such $m$ and we are left with a sequence of density at least $1-3 / G$.

Next take $D=3$ in Lemma 4.2 , and choose $E$ sufficiently large so that

$$
|\{n \in \mathcal{N}(T):|\mathcal{N}(T) \cap[n-D, n+D]|>E+1\}| \leq T / G
$$

removing also these elements we are left with a set of density at least $1-4 / G$.

Now, for $m \in \mathcal{N}^{\prime}$ we have the following:

1. $\left|\lambda_{m}-m\right| \leq G$,

2. $|\{n \in \mathcal{N}: 0<|m-n| \leq 3\}| \leq E$

3. $\sum_{n \in \mathcal{N}:|m-n|>3} \frac{1}{(m-n)^{2}} \leq F$

Thus, if we consider pure momentum observables, and given $n \in \mathcal{N}$ we let $\mu_{n}$ denote the measure on the unit circle consisting of four delta measures (corresponding to lattice points lying on a circle of radius $\sqrt{n}$ ), we find that the measure - not necessarily a probability measure since we have not yet normalized - associated with $G_{\lambda_{m}}$ is given by

$$
\sum_{n \in \mathcal{N}} \frac{\mu_{n}}{\left(n-\lambda_{m}\right)^{2}}=\frac{\mu_{m}}{\left(m-\lambda_{m}\right)^{2}}+\sum_{n \in \mathcal{N}: 0<|n-m| \leq 3} \frac{\mu_{n}}{\left(n-\lambda_{m}\right)^{2}}+\sum_{n \in \mathcal{N}:|n-m|>3} \frac{\mu_{n}}{\left(n-\lambda_{m}\right)^{2}}
$$

where the first term is $\gg 1 / G^{2}$, the second sum has at most $E$ terms, and the last sum is $\ll F$. In particular, for $G$ fixed the mass contribution from the first two terms

$$
\frac{\mu_{m}}{\left(m-\lambda_{m}\right)^{2}}+\sum_{n \in \mathcal{N}: 0<|n-m| \leq 3} \frac{\mu_{n}}{\left(n-\lambda_{m}\right)^{2}}
$$

is uniformly bounded from below, and the number of terms in the sum is uniformly bounded from above. Hence the finite sum

$$
\sum_{n \in \mathcal{N}:|n-m| \leq 3} \frac{\mu_{n}}{\left(n-\lambda_{m}\right)^{2}}
$$

carries mass uniformly bounded from below; after normalizing so that we obtain a probability measure, we find that the normalized measure will have a positive proportion of its mass on a finite number of points. 


\section{A Dirichlet boundary conditions}

In Šeba's original paper [18] the author considers an irrational rectangle $D$ with a delta potential placed in the interior of $D$ and Dirichlet boundary conditions. The setting of the torus has the advantage that calculations are much simplified because of translation invariance, i. e. the position of the potential is not important. The subject of this appendix is to illustrate how our proof can easily be modified for this setting. A modification would work in the analogous and correspond to a different character in the Fourier representation of the eigenfunctions.

\section{A.1 The spectrum and eigenfunctions}

Let $D=[0,2 \pi a] \times[0,2 \pi / a], a^{4} \notin \mathbb{Q}$ diophantine. Let $z \in$ int $D$. We study the self-adjoint extensions of the restricted Dirichlet Laplacian $-\left.\Delta\right|_{D_{0}}$, where $D_{0}=\left\{f \in C_{c}^{\infty}(D \backslash\{z\}) \mid\right.$ $\left.\left.f\right|_{\partial D}=0\right\}$. This operator has deficiency indices $(1,1)$ and we denote the one parameter family of self-adjoint extensions by $\left\{-\Delta_{\varphi}^{D}\right\}_{\varphi \in(-\pi, \pi]}$.

The eigenfunctions of $-\Delta_{\varphi}^{D}$ are given by the Green's functions

$$
G_{\lambda}^{D}(x)=\sum_{\substack{\xi \in \mathcal{L} \\ \xi_{1}, \xi_{2}>0}} \frac{\psi_{\xi}(x) \overline{\psi_{\xi}(z)}}{|\xi|^{2}-\lambda}, \quad \psi_{\xi}(x)=\frac{1}{\pi^{2}} \sin \left(\xi_{1} x_{1}\right) \sin \left(\xi_{2} x_{2}\right)
$$

where $\mathcal{L}=\mathbb{Z}(1 / a, 0) \oplus \mathbb{Z}(0, a)$. The eigenvalues are solutions of the equation

$$
\sum_{\substack{\xi \in \mathcal{L} \\ \xi 1, \xi_{2}>0}}\left|\psi_{\xi}(z)\right|^{2}\left\{\frac{1}{|\xi|^{2}-\lambda}-\frac{1}{|\xi|^{2}+1}\right\}=C_{\mathcal{L}} \tan \left(\frac{\varphi}{2}\right)
$$

where $C_{\mathcal{L}}=\sum_{\xi \in \mathcal{L}} \frac{1}{|\xi|^{4}+1}$.

\section{A.2 Modification of the proof of Theorem 1.4}

We can rewrite the function $G_{\lambda}^{D}$ as

$$
\sum_{\xi \in \mathcal{L}} \frac{\chi(\xi) \overline{\psi_{\xi}(z)} e^{\mathrm{i}\langle x, \xi\rangle}}{|\xi|^{2}-\lambda}, \quad \text { where } \chi(\xi)=\operatorname{sgn}\left(\xi_{1}\right) \operatorname{sgn}\left(\xi_{2}\right)
$$

and

$$
\operatorname{sgn}(x)= \begin{cases}1, & \text { if } x>0 \\ 0, & \text { if } x=0 \\ -1, & \text { if } x<0\end{cases}
$$


Proof. To see this, first of all define, given $\xi=\left(\xi_{1}, \xi_{2}\right), \xi_{1}, \xi_{2}>0$, define $\bar{\xi}=\left(\xi_{1},-\xi_{2}\right)$. We may expand the Laplacian eigenfunctions into complex exponentials

$$
\begin{aligned}
\psi_{\xi}(x) & =\frac{1}{\pi^{2}} \sin \left(\xi_{1} x_{1}\right) \sin \left(\xi_{2} x_{2}\right) \\
& =-\frac{1}{4 \pi^{2}}\left(e^{\mathrm{i} \xi_{1} x_{1}}-e^{-\mathrm{i} \xi_{1} x_{1}}\right)\left(e^{\mathrm{i} \xi_{2} x_{2}}-e^{-\mathrm{i} \xi_{2} x_{2}}\right) \\
& =-\frac{1}{4 \pi^{2}} \sum_{\eta=\xi,-\xi, \bar{\xi},-\bar{\xi}} \chi(\eta) e^{\mathrm{i}\langle\eta, x\rangle} .
\end{aligned}
$$

Hence, we obtain (noting $\psi_{\xi}=0$ if $\xi_{1} \xi_{2}=0$ )

$$
\begin{aligned}
G_{\lambda}^{D}(x) & =\sum_{\substack{\xi \in \mathcal{L} \\
\xi_{1}, \xi_{2}>0}} \frac{\psi_{\xi}(x) \overline{\psi_{\xi}(z)}}{|\xi|^{2}-\lambda} \\
& =-\frac{1}{4 \pi^{2}} \sum_{\xi \in \mathcal{L}} \frac{\chi(\xi) \overline{\psi_{\xi}(z)} e^{\mathrm{i}\langle\xi, x\rangle}}{|\xi|^{2}-\lambda}
\end{aligned}
$$

Let $g_{\lambda}^{D}=G_{\lambda}^{D} /\left\|G_{\lambda}^{D}\right\|_{2}$ and define

$$
d(\xi):=\frac{\chi(\xi) \overline{\psi_{\xi}(z)}}{|\xi|^{2}-\lambda} .
$$

We then obtain for the matrix element of a pure momentum monomial $e_{0, k}$ that

$$
\begin{aligned}
\left\langle O p\left(e_{0, k}\right) g_{\lambda}^{D}, g_{\lambda}^{D}\right\rangle & =\frac{\sum_{\xi \in \mathcal{L} \backslash\{0\}}(\tilde{\xi} /|\xi|)^{k}|d(\xi)|^{2}+|d(0)|^{2}}{\sum_{\xi \in \mathcal{L}_{0}}|d(\xi)|^{2}} \\
& =\frac{\sum_{n \in \mathcal{N}} \frac{\delta_{n} w_{k}(n)}{(n-\lambda)^{2}}}{\sum_{n \in \mathcal{N}} \frac{\delta_{n} \cdot r(n)}{(n-\lambda)^{2}}}
\end{aligned}
$$

where $\delta_{n}=\left|\psi_{\xi(n)}(z)\right|^{2}$ and $\xi(n) \in \mathcal{L}$ is the lattice vector which solves the equation $|\xi|^{2}=n, \xi_{1}, \xi_{2} \geq 0$, and

$$
w_{k}(n)=\sum_{\substack{\xi \in \mathcal{L} \\|\xi|^{2}=n}}\left(\frac{\tilde{\xi}}{|\xi|}\right)^{k} .
$$

Assumption: Suppose that the position $z \in \operatorname{int} D$ is "generic' 7 in the sense that $z_{1} a, z_{2} / a \notin \mathbb{Q}$. This ensures that $\delta_{n}>0$ for all $n \in \mathcal{N}$.

Clumping: The proof of [17] can easily be modified for rectangles with Dirichlet boundary conditions. Thus we obtain the analogue of Theorem 3.2 for the operator $-\Delta_{\varphi}^{D}$.

\footnotetext{
${ }^{7}$ In the case of rational coordinates there will be a positive proportion of eigenvalues whose eigenfunctions vanish at the position of the scatterer and therefore do not feel its effect. In the generic case of irrational coordinates all eigenfunctions feel the effect of the scatterer, therefore there are only "new" eigenfunctions.
} 
In order to construct localized semiclassical measures we pick a subsequence $n \in$ $\mathcal{N}^{\prime} \subset \mathcal{N}$, of density $1-\epsilon$ for any $\epsilon>0$, such that $\liminf _{n} \delta_{n}=\delta>08$. For mixed monomials $e_{\zeta, k}$ we may now apply exactly the same argument as in the proof of Theorem 2.1, to see that $\lim _{n \in \mathcal{N}^{\prime}}\left\langle O p\left(e_{\zeta, k}\right) g_{\lambda_{n}}^{D}, g_{\lambda_{n}}^{D}\right\rangle=0$. The analogue of Proposition 3.1 is again proved in exactly the same way as above. Hence the analogue of Theorem 1.4 for diophantine rectangles with Dirichlet boundary conditions follows.

Remark 3. In a similar fashion we can prove the result also for the strong coupling limit (see section 4), where within a subsequence of positive density the eigenfunctions have positive mass on a finite number of Dirac masses in momentum space.

\section{References}

[1] M. Berry, Regular and irregular semiclassical wave functions, J. Phys. A 10, 208391, 1977.

[2] M. Berry, M. Tabor, Level clustering in the regular spectrum, Proc. Royal Soc. Lond. A 356, 375-394, 1977.

[3] G. Berkolaiko, J. P. Keating and B. Winn, No quantum ergodicity for star graphs, Comm. Math. Phys. Vol. 250, 259-285, 2004.

[4] S. de Bièvre, F. Faure, S. Nonnenmacher, Scarred eigenstates for quantum cat maps of minimal periods, Comm. Math. Phys. 239, 449-492, 2003.

[5] E. Bogomolny, U. Gerland and C. Schmit, Singular Statistics, Phys. Rev. E, Vol. 63, No. 3, 2001.

[6] E. Bogomolny, C. Schmit, Structure of Wave Functions of Pseudointegrable Billiards, Phys. Rev. Lett. 92 (2004), No. 24, 244102.

[7] J. Bourgain, A remark on quantum ergodicity for CAT maps. Geometric aspects of functional analysis, 8998, Lecture Notes in Math., 1910, Springer, Berlin, 2007.

[8] Y. Colin de Verdière, Ergodicité et fonctions propres du laplacien. Comm. Math. Phys. Vol. 102, No. 3, 497-502, 1985.

[9] A. Eskin, G. Margulis and S. Mozes, Quadratic forms of signature (2,2) and eigenvalue spacings on rectangular tori. Ann. of Math. (2) 161, no. 2, 679-725, 2005.

\footnotetext{
${ }^{8}$ Such a sequence may easily be constructed by noticing that the set $\left\{\left(z_{1} \xi_{1}, z_{2} \xi_{2}\right)\right\}_{\xi \in \mathcal{L}}$ equidistributes modulo $[0,2 \pi]^{2}$ if $z$ is generic in the above sense.
} 
[10] F. A. Berezin and L. D. Faddeev, Remark on the Schrödinger equation with a singular potential, Dokl. Akad. Nauk SSSR 137, 1011-1014, 1961 (Russian); English translation: Soviet Mathematics 2, 372-375, 1961.

[11] A. Hassell, Ergodic billiards that are not quantum unique ergodic. With an appendix by A. Hassell and L. Hillairet. Ann. of Math. (2), no. 171, 605 - 618, 2010.

[12] J. P. Keating, J. Marklof and B. Winn, Localised eigenfunctions in Šeba billiards. J. Math. Phys. 51, no. 062101.

[13] D. Kelmer, Arithmetic quantum unique ergodicity for symplectic linear maps of the multidimensional torus. Ann. of Math. (2) 171 (2010), no. 2, 815879.

[14] R. de L. Kronig and W. G. Penney, Quantum Mechanics of Electrons in Crystal Lattices. Proceedings of the Royal Society of London. Series A, Vol. 130, No. 814, 499-513, 1931.

[15] P. Kurlberg, H. Ueberschär, Quantum ergodicity for point scatterers on arithmetic tori, Geom. Funct. Anal. 24, No. 5, 1565-90, 2014.

[16] Z. Rudnick, H. Ueberschär, Statistics of wave functions for a point scatterer on the torus. Comm. Math. Phys., Vol. 316, No. 3, 763-782, 2012.

[17] Z. Rudnick, H. Ueberschär, On the eigenvalue spacing distribution for a point scatterer on a flat torus. Annales Henri Poincaré Vol. 15, No. 1, 1-27, 2014.

[18] P. Šeba, Wave chaos in singular quantum billiard. Phys. Rev. Lett. 64, 1855-1858, 1990.

[19] P. Šeba, P. Exner, Point interactions in two and three dimensions as models of small scatterers. Physics Letters A 222, 1-4, 1996.

[20] T. Shigehara, Conditions for the appearance of wave chaos in quantum singular systems with a pointlike scatterer, Phys. Rev. E, Vol. 50, No. 6, 1994.

[21] T. Shigehara, T. Cheon, Wave chaos in quantum billiards with a small but finite-size scatterer, Phys. Rev. E, Vol. 54, No. 2, 1321-1331, 1996.

[22] T. Shigehara, T. Cheon, Spectral properties of three-dimensional quantum billiards with a pointlike scatterer, Phys. Rev. E 55, 6832-684, 1997.

[23] T. Shigehara, H. Mizoguchi, T. Mishima, T. Cheon, Chaos Induced by Quantization, 1998. 
[24] T. Tudorovskiy, U. Kuhl, H-J. Stöckmann, Singular statistics revised, New J. Phys. 12, 2010. 